\title{
Volle Pipeline beim kastrationsresistenten Prostatakarzinom
}

n den letzten Jahren wurden zahlreiche

Substanzen zur Behandlung verschiedener Stadien des kastrationsresistenten Prostatakarzinoms (CRPC) zugelassen. Die Pharmafirmen forschen weiter, sodass bald noch mehr neue Medikamente dazukommen könnten.

Ein möglicher Kandidat ist der selektive nicht steoridale 17,20-Lyase-Inhibitor Orteronel (ortl, TAK-700), mit dem grundsätzlich eine Steroid-freie Gabe möglich ist. Sean Moran, Cambridge/USA, präsentierte eine kleine Studie mit 39 Prostatakarzinompatienten mit einem durchschnittlichen PSA-Wert von 12,1 ng/ml. Nach drei Monaten Behandlung mit Orteronel konnte der PSA-Wert bei $93 \%$ der Patienten reduziert werden (im Schnitt um $83 \%$ ), bei $16 \%$ unter $0,2 \mathrm{ng} / \mathrm{ml}$.

Sipuleucel-T ist ein „Impfstoff“ mit dendritischen Zellen, die aus dem Blut von betroffenen Prostatakarzinompatienten gewonnen werden. Er soll die körpereigene Immunabwehr gegen die Krebszellen stärken und ist in den USA für die Behandlung von asymptomatischen Patienten mit metastasiertem CRPC zugelassen. Dr. Robert Sims, Seattle/USA, stellte eine Studie mit sieben Patienten vor, die bereits in der $\mathrm{Zu}$ lassungsstudie PROTECT mit Sipuleucel-T behandelt worden waren und nun einen Tumorprogress hatten. Die Patienten erhielten im Mittel 8,6 Jahre nach der letzten Infusion eine erneute Sipuleucel-T-Dosis. Die Immunantwort fiel bei allen Patienten wesentlich stärker aus, was für ein Langzeit-Immungedächtnis spricht.

GTx-758, ein Estrogenrezeptor-alphaAgonist, könnte in der Androgendeprivation eingesetzt werden. In einer Phase-
II-Studie wurden die Dosen $1.000 \mathrm{mg} / \mathrm{d}$ und $2.000 \mathrm{mg} / \mathrm{d}$ mit einem ViermonatsDepot Leuprorelin verglichen. Mit beiden GTx-758-Formulierungen konnten PSA, freies und Serumtestosteron signifikant deutlicher gesenkt werden als mit dem GnRH-Analogon. Zudem hatten binnen 90 Behandlungstagen wesentlicher weniger GTx-758-Patienten Hitzewallungen (18,8\% bzw. $5,6 \%)$ als unter Leuprorelin (80,9\%). Aufgrund von venösen Thromboembolien (VTE) in den GTx-758Gruppen wurde die Studie jedoch frühzeitig gestoppt. Derzeit werden GTx-758Dosen von 125 - $500 \mathrm{mg}$ untersucht, um zu testen, ob sich die antihormonelle Wirkung auch ohne erhöhtes VTE-Risiko erreichen lässt.

Sebastian Lux

P100, P102, P103

\section{ACHTUNG:}

\section{Hier muss der Dummy durch eine Anzeige ersetzt werden !!}

